Portland State University

PDXScholar

$5-22-1980$

\title{
Effective Force Constant Ratios: Iron in Iridium and Rhodium
}

Dennis Munsterman

Portland State University

Follow this and additional works at: https://pdxscholar.library.pdx.edu/open_access_etds

Part of the Atomic, Molecular and Optical Physics Commons Let us know how access to this document benefits you.

\section{Recommended Citation}

Munsterman, Dennis, "Effective Force Constant Ratios: Iron in Iridium and Rhodium" (1980). Dissertations and Theses. Paper 2983.

https://doi.org/10.15760/etd.2980

This Thesis is brought to you for free and open access. It has been accepted for inclusion in Dissertations and Theses by an authorized administrator of PDXScholar. Please contact us if we can make this document more accessible: pdxscholar@pdx.edu. 
AN ABSTRACT OF THE THESIS OF Dennis Munsterman for the Master of Science in Physics presented May 22, 1980.

Title: Effective Force Constant Ratios: Iron In Iridium and Rhodium.

APPROVED BY MEMBERS OF THE THESIS COMMITTEE:

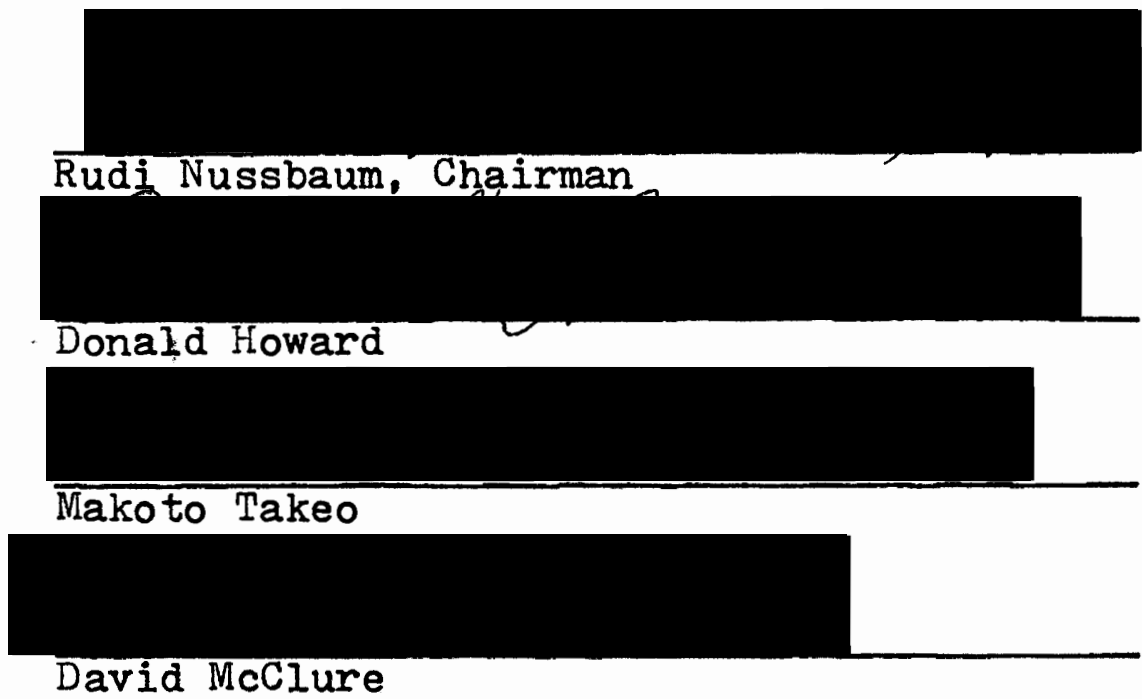

Classical methods of analyzing heat capacity data for the characteristic moments of the frequency distribution are applied to iridium and rhodium. Impurity moments are determined from high and low temperature $f$ values. These moments are combined by modern theory to estimate the magnitude of the host-host to host-impurity force constant ratio. Ratios of the various host moments are also examined. 
EFFECTIVE FORCE CONSTANT RATIOS,

IRON IN IRIDIUM AND RHODIUII

by

DENNIS MUNSTERMAN

A thesis submitted in partial fulfillment of the requirements for the degree of

MASTER OF SCIENCE

in

PHYSICS

Portland State University

1980 
TO THE OFFICE OF GRADUATE STUDIES AND RESEARCL:

The members of the Committe approve the thesis of Dennis Munsterman presented May 22, 1980.
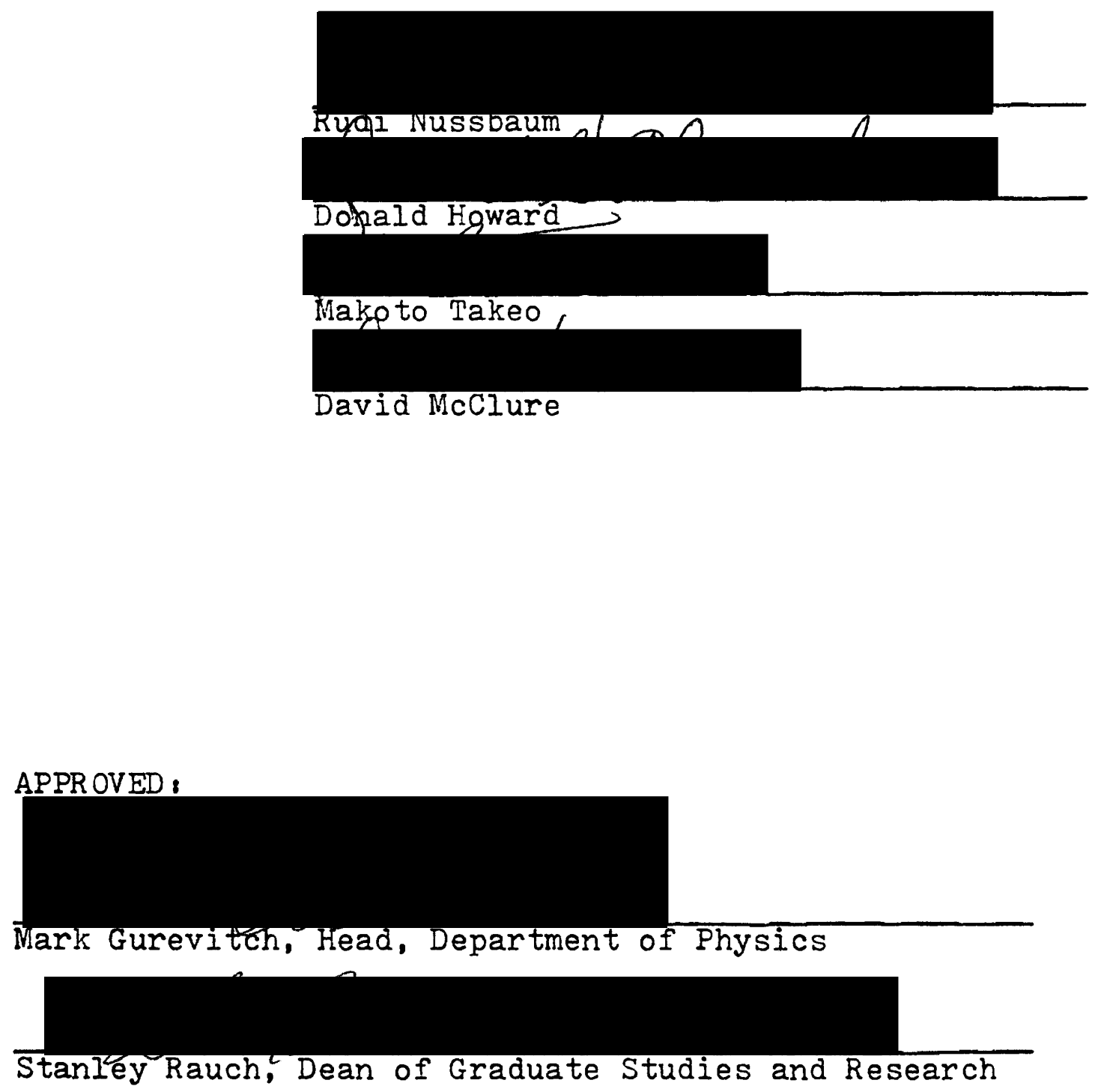


\section{ACKN OWLEDGMENTS}

For their patience and help. I thank Drs. Rudi Nussbaum and Donald Howard. To Drs. Phillip Gold, Mark Gurevitch, and Makoto Takeo I extend my appreciation for helping me to return to those things I left as a child. To Mary Kelley for the sacrifices she had to make to electricity and magnetism or mechanics, and Katherine Iee for the motive force, I extend my thanks. 


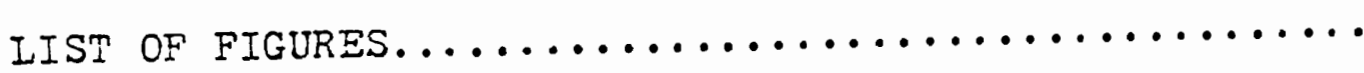

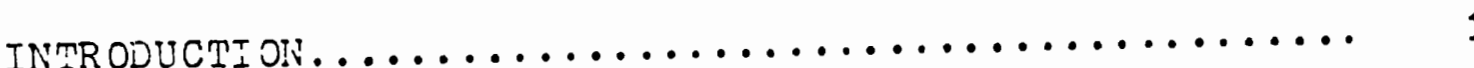

HOST WHONENTS FROH HEAT CAPACITY DATA............ 3

Heat Capacity........................... 3

i.oment Analysis of heat Capacity.............. 9

Low Temperature Region................. 9

High Temperature Region................ 12

Moments and the Frequency Distribution..... 17

Intermediate Temperature Region.......... 20

EXPER I.ENTAL PROCEDURE....................... 23

Preparation of fiössbauer sources............. 23

Recoil-Free Fraction.................... 25

IMPURITY NONENTS AND EFFECTIVE FORCE CONSTANT RATIOS.. 31

DISCUSSION............................... 35

BIPIIOGRAPFY............................... 38 
LIST OF TABLES

TABLE

PAGE

I

PHYSICAI CONSTANTS

I HOST MONENTS AND MOMENT RATIOS............. 14

II VELOCITY SPECTRUM, IRIDIUN.............. 26

IV RECOIL-FREE FRACTION $f$, AND IOG ........... 30

$\mathrm{V}$ IMPURITY MOMENTS AND EFFECTIVE FORCE CONSTANT

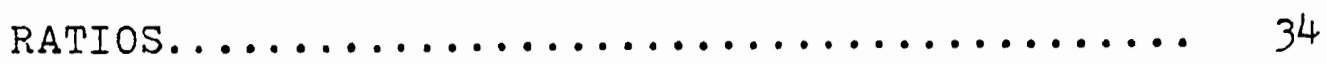




\section{LIST OF FIGURES}

FIGURE

PAGE

1 Graph of $\mathrm{C}_{\mathrm{V}} / \mathrm{T}^{3}$ versus $\mathrm{T}^{2}$ for several values of $\gamma^{\prime} \ldots \ldots \ldots \ldots \ldots \ldots \ldots \ldots \ldots \ldots \ldots \ldots$

2 Graph of $\theta$ versus temperature for iridium..... 15

3 Graph of $\theta^{2}\left(V_{0}\right)$ versus $1 / T^{2}$ for iridium....... 16

4 Generalized frequency distribution for a metal. 18 


\section{INTRODUCTION}

The studies directed to the properties of materials have long focused on the need to describe the forces between atoms in the crystalline lattice. The nature and magnitude of the forces between adjacent atoms and for those further away has been difficult to study experimentally.

Recently, however, both theoretical ${ }^{1}$ and experimental ${ }^{2}$ procedures have been developed to allow a closer look at the nature of the forces between atoms in the lattice. The application of the Born-von Karman analysis to neutron dispersion relations has allowed accurate determination of the elastic constants of pure metals. Neutron dispersion curves are not available for all metals, however, and in those cases, an analysis of the heat capacity data leads to a determination of the moments of the frequency distribution. ${ }^{2}$ Impurity moments can also be determined from the mean squared displacement of substitutional impurity atoms from the recoil-free fraction $f$ of the Mössbauer effect. ${ }^{1}$ By combining moments of the host and impurity frequency distribution with a reasonable physical model of the lattice (with an impurity present) ${ }^{3}$, it is now possible to derive a ratio of the host-host to host-impurity restoring forces. 16 This thesis will treat the method by which the host moments can be derived from heat capacity data, how the 
impurity moments can be deduced from Mössbauer measurements at low and high temperatures, and the effective force constant ratio derived from them, as applied to isolated iron impurity atoms in an iridium or rhodium lattice. 


\section{HOST MOMENTS FROM HEAT CAPACITY DATA}

Early in this century it became clear that the heat capacity of materials was related to their internal structure.

The theories of lattice vibrations of Einstein and Debye found their testing ground in the fundamental relationship between heat capacity and temperature. It was observed that the frequency distribution could be related to the heat capacity in the low to intermediate temperature range if corrections for volume expansions with temperature could be made.

\section{HEAT CAPACITY}

The heat capacity of a material is defined to be the amount of heat per mole required to raise the temperature one degree. This is expressed in terms of the heat absorbed by the system $d Q$ as

$$
\mathrm{C}=\mathrm{dQ} / \mathrm{dT},
$$

where $d Q$ is the amount of heat absorbed by one mole of the material. The first law of thermodynamics allows one to write $d Q$ as

$$
d Q=d U+p d V=d H-V d p,
$$

where we have used the relation $\mathrm{H}=\mathrm{U}+\mathrm{pV}$, and the terms 
are as follows (on a molar basis); $U$ is the internal energy, $H$ is the enthalpy, $V$ is the molar volume, and $p$ is the pressure.

In order to define the process by which the material absorbs heat in a thermodynamic sense, we must specify which of the variables will remain constant as the system changes with temperature. If volume is constant in eqn. (2), then

$$
C_{v}=(\partial U / \partial T)_{v} \text {, }
$$

and if the pressure remains constant,

$$
\mathrm{C}_{\mathrm{p}}=(\partial \mathrm{H} / \partial \mathrm{T})_{\mathrm{p}} \text {. }
$$

The heat capacity at constant pressure is found experimentally, but the heat capacity at constant volume is the quantity directly related to the lattice vibrations in models. The relationship between $\mathrm{C}_{\mathrm{p}}$ and $\mathrm{C}_{\mathrm{v}}$ is as follows.

First, we note that in a reversible process,

$$
C_{p}=(\partial Q / \partial T)_{p}=T(\partial S / \partial T)_{p} \text {, }
$$

and

$$
C_{\mathrm{v}}=(\partial Q / \partial \mathrm{T})_{\mathrm{v}}=\mathrm{T}(\partial \mathrm{S} / \partial \mathrm{T})_{\mathrm{V}} \text {. }
$$

Then taking the differential of the entropy, $S$, as

$$
d S=(\partial S / \partial T)_{V} d T+(\partial S / \partial V)_{T} d V \text {, }
$$

we find

$$
(\partial S / \partial T)_{p}=(\partial S / \partial T)_{V}+(\partial S / \partial V)_{T}(\partial S / \partial T)_{p} \cdot
$$

If we now use the Maxwell relation

$$
(\partial \mathrm{S} / \partial \mathrm{V})_{\mathrm{T}}=(\partial \mathrm{p} / \partial \mathrm{T})_{\mathrm{V}}=-(\partial \mathrm{p} / \partial \mathrm{V})_{\mathrm{T}}(\partial \mathrm{V} / \partial \mathrm{T})_{\mathrm{p}},
$$


we find that

$$
\begin{aligned}
& C_{p}-C_{v}=T(\partial p / \partial T)_{v}(\partial V / \partial T)_{p}= \\
& -T(\partial p / \partial V)_{T}(\partial V / \partial T)_{p}^{2}=T V B^{2} / k_{T},
\end{aligned}
$$

where $B=(1 / V)(\partial V / \partial T)_{p}$ is the volume coefficient of thermal expansion, $k_{\mathrm{T}}=-(1 / \mathrm{V})(\partial \mathrm{V} / \partial \mathrm{p})_{\mathrm{T}}$ is the isothermal compressability, and $\mathrm{V}$ is the molar volume. These three are all functions of temperature, but an empirical relation, called the Nernst-Lindemann relation, which includes all three but is constant over temperature, has been observed, 4

$$
\bar{A}=\mathrm{VB}^{2} / \mathrm{k}_{\mathrm{T}} \mathrm{C}_{\mathrm{p}}^{2}
$$

This allows one to write the dilation term of the heat capacity as

$$
\mathrm{C}_{\mathrm{p}}-\mathrm{C}_{\mathrm{v}}=\mathrm{AC}_{\mathrm{p}}^{2} \mathrm{~T}
$$

In a metal, the heat capacity can be separated into two contributions, one from the vibrations of the lattice, and the other from the conduction electrons. The latter is well known from the theory of metals to be linear with temperature (for $\mathrm{T} \ll \mathrm{T}_{\text {Fermi }}$ ), i.e.

$$
c_{v}=c_{v}^{l}+c_{v}^{e}=c_{v}^{l}+\gamma_{e}^{T} \text {, }
$$

where $\gamma_{e}$ is the coefficient of the electronic component. This allows one to express the theoretically important lattice heat capacity in terms of the two experimentally derived quantities $C_{p}$ and $\gamma_{e}$,

$$
C_{v}^{I}=C_{p}\left(1-A_{p} T\right)-\gamma_{e} T \text {. }
$$

As can be seen in Table I the values of $\bar{A}$ are several orders of magnitude below $\gamma_{e}$. This may not be true for all 
TABLE I

PHYSICAI CONSTANTS

IRIDIUM

RHODIUM

\begin{tabular}{lllllll} 
Temp & $\alpha \times 10^{6}$ & $\begin{array}{c}\Delta l / 1 \\
(\%)\end{array}$ & $\begin{array}{c}\bar{A} \times 10^{6} \\
\mathrm{~m} / \mathrm{cal}\end{array}$ & $\alpha \times 10^{6}$ & $\begin{array}{c}\Delta I / 1 \\
(\%)\end{array}$ & $\begin{array}{l}\bar{A} \times 10^{6} \\
\mathrm{~m} / \mathrm{cal}\end{array}$ \\
\hline 10 & .01 & - & 3.4 & .01 & - & 2.2 \\
20 & .09 & - & 5.9 & .10 & - & 11. \\
50 & 1.8 & - & 7.0 & 1.8 & - & 11. \\
100 & 4.4 & .019 & 7.5 & 5.0 & .014 & 9.4 \\
200 & 5.9 & .073 & 7.4 & 7.3 & .076 & 9.0 \\
293 & 6.4 & .130 & 7.6 & 8.2 & .149 & 9.4 \\
400 & - & .200 & - & - & .240 & - \\
500 & - & .269 & - & - & .331 & - \\
600 & - & .342 & - & - & .427 & - \\
\hline$\gamma^{\prime}$ & & $(.765 \pm .024) \times 10^{-3}$ & $(1.111 \pm .036) \times 10^{-3}$
\end{tabular}

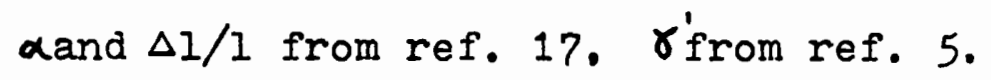


metals, but for the fcc, refractory metals considered here, $\mathbb{A}$ can be incorporated into the electronic coefficient. This was simply done by Furukawa et.al. 5 on the heat capacity data used here, since their numerical "smoothing" technique allows them to derive an effective electronic coefficient $\gamma$. by forcing the relation between the three terms into the form

$$
c_{v}^{I}=c_{p}-\gamma^{\prime T}
$$

The quasiharmonic correction to the characteristic Debye temperature, $\theta$, is based on the assumption that the characteristic frequencies change as the volume changes with temperature. This will become more apparent from the Debye equation of state, which we derive as follows. 6 we can express the Helmholtz free energy of the crystal as

$$
A=U-T S \text {, }
$$

and therefore

$$
(\partial \mathrm{A} / \partial \mathrm{T})_{\mathrm{V}}=-\mathrm{S} .
$$

so that the internal energy can be written as

$$
U=A-T(\partial A / \partial T)_{V}=[\partial(A / T) \partial(1 / T)]_{V} \text {. }
$$

Also the pressure of the solid can be derived from the Helmholtz free energy as

$$
\mathrm{p}=-(\partial \mathrm{A} / \partial \mathrm{V})_{\mathrm{T}} \text {. }
$$

If we assume that the Helmholtz energy is a function of temperature and volume only, then we may write

$$
A=U_{0}(V)+A_{D}(T, V) \text {. }
$$

where $U_{0}$ is the internal energy at $0^{\circ} \mathrm{K}$, and $A_{D}$ is the 
free energy of the lattice.

For the Debye model we may assume that the dependence of the free energy on the volume is given by specifying the dependence of the Debye temperature $\theta$ on V. Therefore, from eqns, (19) and (20),

$$
\mathrm{p}=-\left(\partial U_{0} / \partial V\right)_{T}-\left[\left(\partial A_{D} / \partial \theta\right)(\partial \theta / \partial V)\right]_{T} \cdot
$$

Since in the Debye model the internal energy $U_{D}$ is composed of temperature multiplied times a function of $\theta / T$, by eqn. (18) the Helmholtz free energy must be of the same form,

$$
A_{D}=T \cdot f(\theta / T)
$$

therefore

$$
\partial A_{D} / \partial \theta=(1 / \theta) \partial\left(A_{D}\right) / \partial(1 / T)=U_{D} / \theta \text {. }
$$

Substituting eqn. (23) in eqn. (21) we arrive at the Debye equation of state,

$$
p=-\left(\partial U_{0} / \partial V\right)+\gamma_{G} U_{D} / V
$$

where

$$
\gamma_{\mathrm{G}}=-\mathrm{V} / \theta \mathrm{d} \theta / \mathrm{dV}=-\mathrm{d} \ln \theta / \mathrm{d} \ln \mathrm{V},
$$

is called the Grüneisen constant. For temperatures

low enough for the Debye model to hold, we may reduce the Debje temperature $\theta(V)$ at the temperature of the experiment to the Debye temperature at constant volure (i.e. $0^{\circ} \mathrm{K}$ ), by using eqn (25) to find

$$
\theta\left(v_{0}\right) / \theta(v)=\left(v / v_{0}\right)^{\gamma_{G}}
$$

where $V$ is the volume of the crystal at the temperature of the experiment and $V_{0}$ is the volume at zero degree 
Kelvin.

In using the Gruneisen constant, it is necessary to use the values of $B, k_{T}$, and $c_{v}$ characteristic of the temperature at which the heat capacity was evaluated to give the $\theta(V)$ which is to be reduced to $\theta\left(V_{0}\right)$.

Observing that for a fcc crystal

$$
\mathrm{v}_{0}=\mathrm{V}-\Delta \mathrm{V}=\mathrm{V}\left(1-3 \mathrm{I}^{2} \Delta \mathrm{I} / \mathrm{I}^{3}\right)=\mathrm{V}(1-3 \Delta \mathrm{I} / \mathrm{l})
$$

we find from eqn. (26),

$$
\left[\theta\left(v_{0}\right)\right]^{2}=[\theta(v)]^{2}\left[1 /(1-(3 \Delta I / I)]^{2 \gamma_{G}}\right.
$$

This correction can be significant, but is less than .2 percent for Ir and Rh at 100 degrees Kelvin but rises to around 2 percent in Pt at $200 \mathrm{~K}$.

\section{MOMENT ANALYSIS OF HEAT CAPACIBY}

Low Temperature Region. At low temperatures, the heat capacity can be expanded in powers of the temperature

$$
c_{v}^{1}=a T^{3}+b T^{5}+c T^{7}+\ldots \text {, }
$$

where $a, b$, and $c$ are constants. We also know that at low temperature the Debye approximation can be used. For temperatures less than about eight percent of the low temperature Debye theta, $\theta$, (i.e. for $\mathrm{T}<\theta / 12$ ), the Debye approximation allows one to write

$$
C_{v}^{1}=\left(4 \pi^{4} / 5\right) 3 R(T / \theta)^{3} \text {. }
$$

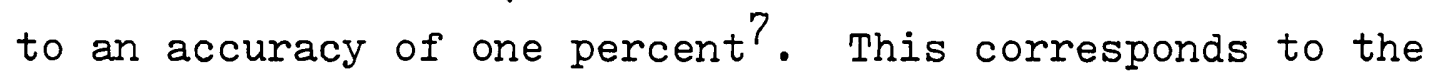
first term in eqn. (29), and a graph of $\mathrm{C}_{\mathrm{v}}^{1} / \mathrm{T}^{3}$ versus $\mathrm{T}^{2}$ will give the value of the low temperature Debye theta 
from the intercept as $\mathrm{T}^{2}$ goes to zero,

$$
\theta_{0}=\left(12 \pi^{4} R / 5 a\right)^{1 / 3} \text {, }
$$

where the intercept a is the coefficient of the cubic term in eqn. (29), and the subscript refers to the temperature at which the Debye temperature is determined.

As a practical matter, it is necessary to take into account the fact that at very low temperatures the measured heat capacity, $c_{p}$ ' is mainly due to the electron gas in the metal. Small variations in the coefficient of the electronic term, $\gamma^{\prime}$, will therefore produce large changes in the deduced lattice heat capacity (fig. 1). It is therefore convenient to allow $\gamma^{\prime}$ to vary slightly around its average value and observe that the lattice heat capacities divided by $\mathrm{T}^{3}$ appear to fan out in the region below approximately eight degrees before diverging almost assymptotically below approximately two degrees Kelvin.

Since we know from the Debye theory that $\mathrm{c}_{\mathrm{V}} / \mathrm{T}^{3}$ is approximately constant in this region, we can extrapolate from the median of this family of curves to zero in order to evaluate $\theta_{0}(f i g, 1)$. Also, we can expand the frequency spectrum at low temperatures as

$$
G(\omega)=\alpha \omega^{2}+\beta \omega^{4}+\gamma \omega^{6}+\ldots .,
$$

in which the coefficients $\alpha, \beta$, and $\gamma$ can be related to the coefficients $a, b$, and $c$ of eqn.(29). Thus the graph of $c_{v} / T^{3}$ versus $T^{2}$ at low temperatures gives some 


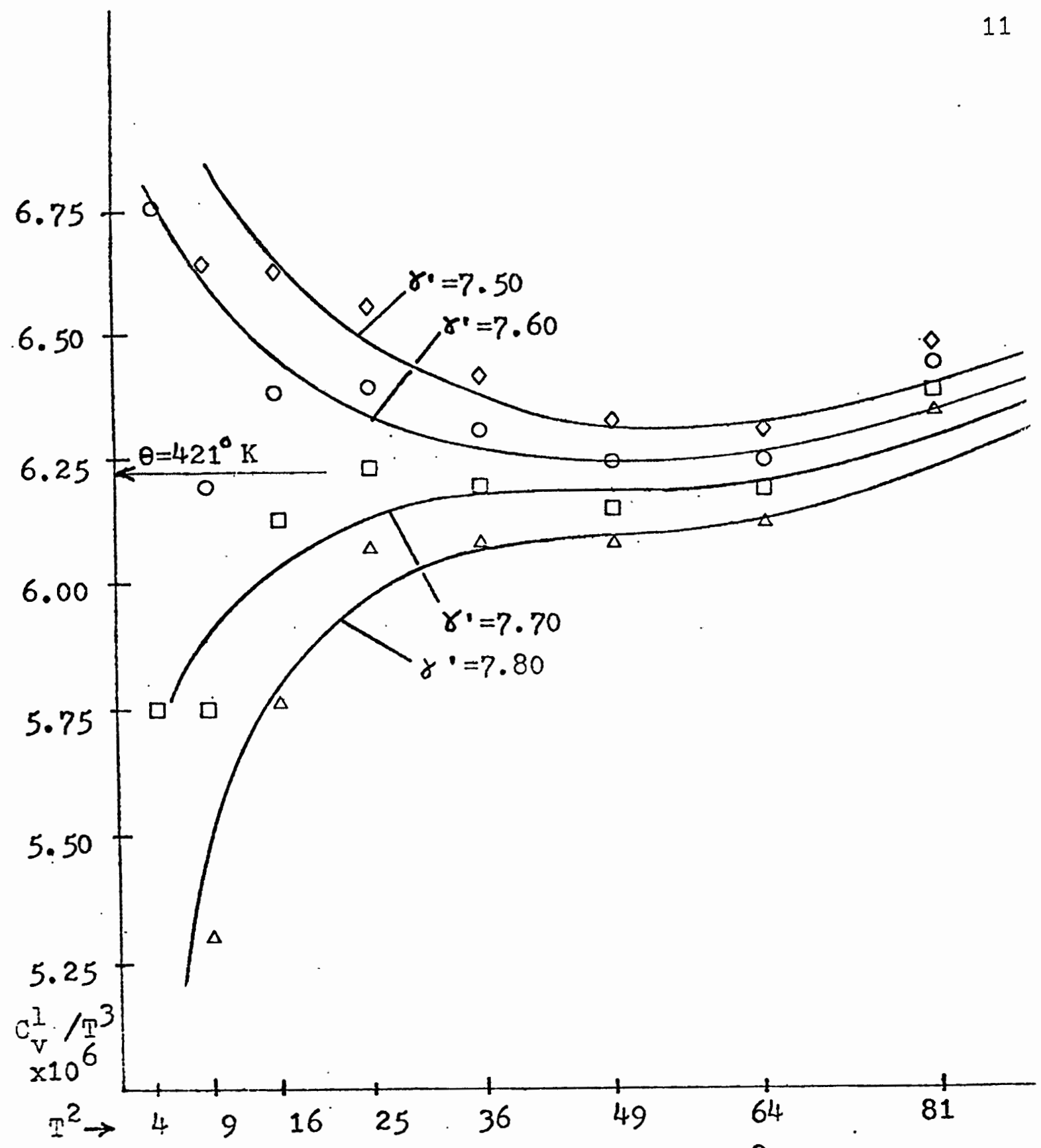

Figure 1. Graph of $\mathrm{C}_{\mathrm{v}}^{1} / \mathrm{T}^{3}$ versus $\mathrm{T}^{2}$ for several 
information about the form of the frequency spectrum at low temperatures.

High Temperature Region. At high temperatures the heat capacity can be expanded as a power series in $1 / T^{2}, 8$ $C_{v}^{I}=3 R\left(1-B_{2} u_{2}^{*} / 2 ! T^{2}+3 B_{4} u_{4}^{*} / 4 ! T^{4}-5 B_{6} u_{6}^{*} / 6 ! T^{6}+\ldots \ldots\right),(33)$ where $B_{2}, B_{4}$, etc are the Bernoulli numbers, and $u_{2}^{*}$, $u_{4}^{*}$, $E \tau c$ are thermal moments derived directly from even moments of the frequency spectrum,

$$
u_{n}^{*}=(n / k)^{n} u(n), \quad u(n)=\int_{0}^{\infty} w^{n} G(\omega) d \omega, u(0)=1, \quad(34)
$$

where $h$ is Planck's constant, $k$ is Boltzmann's constant, and the normalization is consistent with that of the impurity site moments to be discussed later.

The convergence of eqn. (33) is generally slow for temperatures less than $\theta / 2$, and above that temperature anharmonic effects cause considerable problems. However, an equivalent expansion for $\theta$ may be expected to converge rapidly at much lower temperatures ${ }^{9}$. This $\theta$ expansion may be written in the form

$$
\theta^{2}=\theta_{\infty}^{2}\left[1-A\left(\theta_{0} / T\right)^{2}+B\left(\theta_{0} / T\right)^{4}-\ldots\right] \text {, }
$$

where

$$
\begin{aligned}
& \theta_{\infty}=(\hbar / k)(5 u(2) / 3)^{\frac{1}{2}}, \\
& A=(3 / 100)\left[u(4) / u(2)^{2}-25 / 21\right], \\
& B=(1 / 1400)\left[\left(u(6) / u(2)^{3}-125 / 81\right)-100 A\right],
\end{aligned}
$$

These three parameters can be obtained from a least squares curve fit to the values of $\theta^{2}\left(V_{0}\right)$ versus $1 / T^{2}$ obtained from the heat capacity data. They have been 
determined for iridium, and rhodium in the following fashion (table II).

First one takes the heat capacity data and uses them to find values of $\theta / T$ from interpolation in accurate tables of the Debye integral; 10

$$
C_{v}^{1} / 3 R=(T / \theta)^{3} \int_{0}^{x_{m}} e^{x} x^{4} d x /\left(e^{x}-1\right)^{2}
$$

where $x=\hbar \omega / k T$, and $x_{m}=\hbar \omega_{\max } / k T=\theta / T$. This enables one to calculate values of $\theta(V)$.

A plot of $\theta(V)$ versus $T$ (fig. 2) then shows three main regions. In the first region ( $A$ in fig. 2) we see that $\theta$ falls rapidly with temperature to a minimum value. The second region (B) begins with this minimum and includes the slowly rising plateau before the general falling off of $\theta$ at the higher temperatures of the last region (C).

Then by using eqn. (28) on the values of $\theta(V)$ from the second region, and for a few degrees on either side to see the overall relationship, we obtain values of $\theta^{2}\left(v_{0}\right)$ in the quasiharmonic approximation.

By plotting these values of $\theta^{2}\left(v_{0}\right)$ versus $1 / T^{2}$ we observe that the values of $\theta^{2}\left(V_{0}\right)$ show a sharp dip at the minimum value of $\theta$ and fall off rapidly at the upper edge of the middle region (fig. 3). By finding the least squares curve fit to the values of $\theta^{2}\left(v_{0}\right)$ versus $1 / T^{2}$ between their maximum and minimum values, we can find the coefficients in eqn. (35).2 The intercept as $1 / \mathrm{T}^{2}$ goes to zero gives $\Theta_{\infty}^{2}$, the extrapolated high temperature value of 
TABLE II

HOST MOMENTS AND MOMENT RATIOS

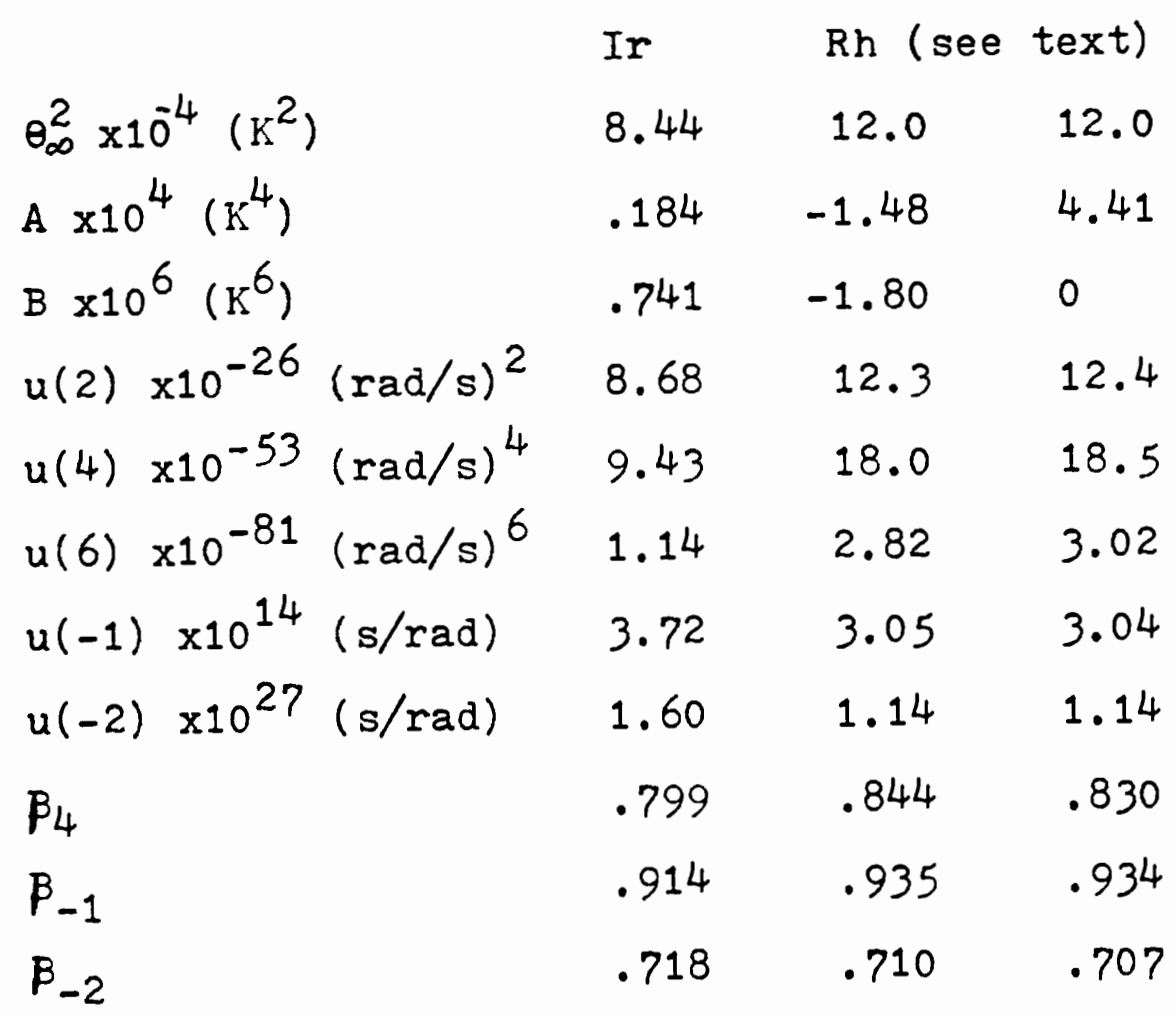




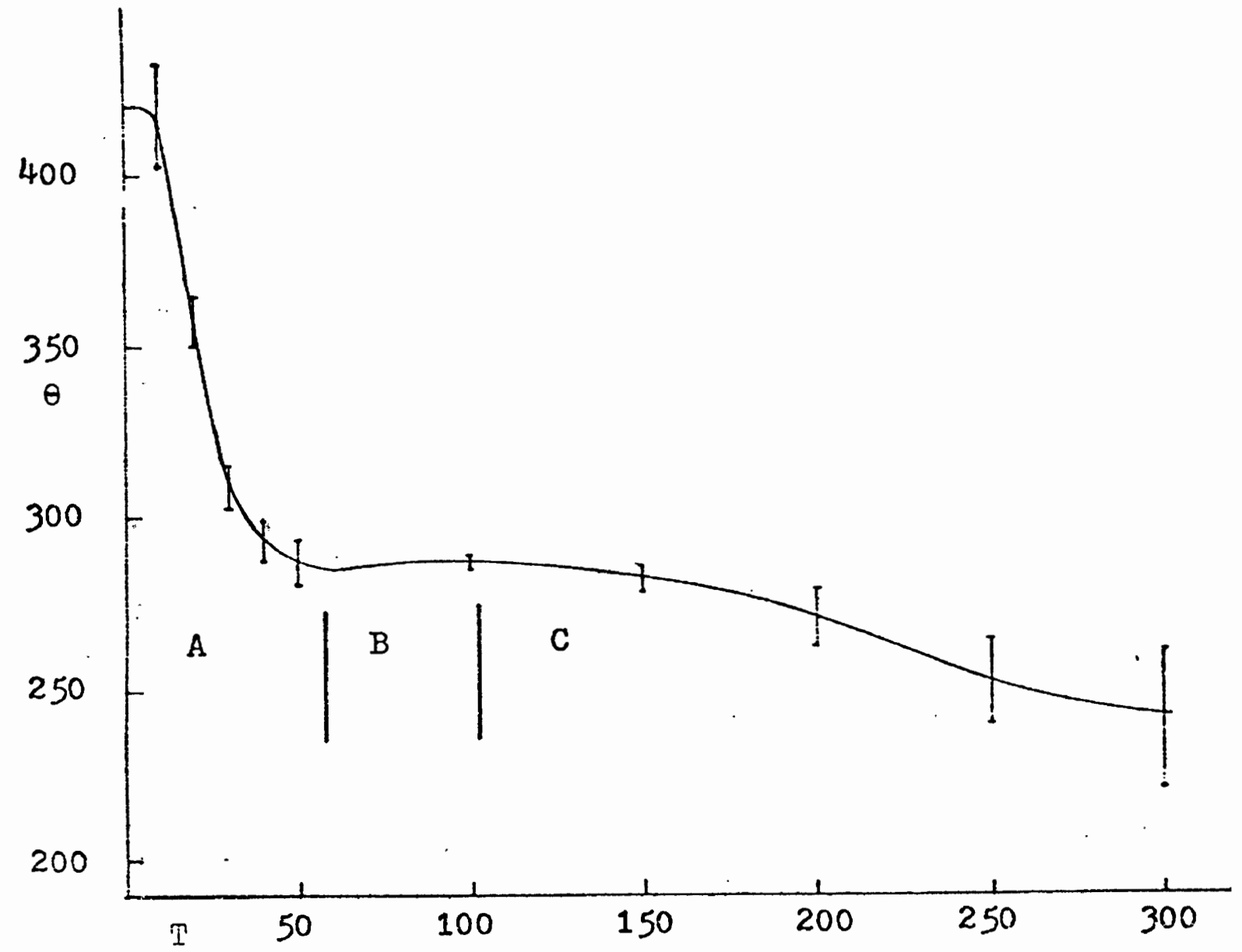

Fisure 2. Graph of $\theta$ versus temperature for iridium. ADove $100 \mathrm{~K}$, the lines correspond to $1 \%$ of $\mathrm{C}_{\mathrm{V}}$, and those below $100 \mathrm{~K}, 10 \%$ of $\mathrm{C}_{\mathrm{V}}$. 


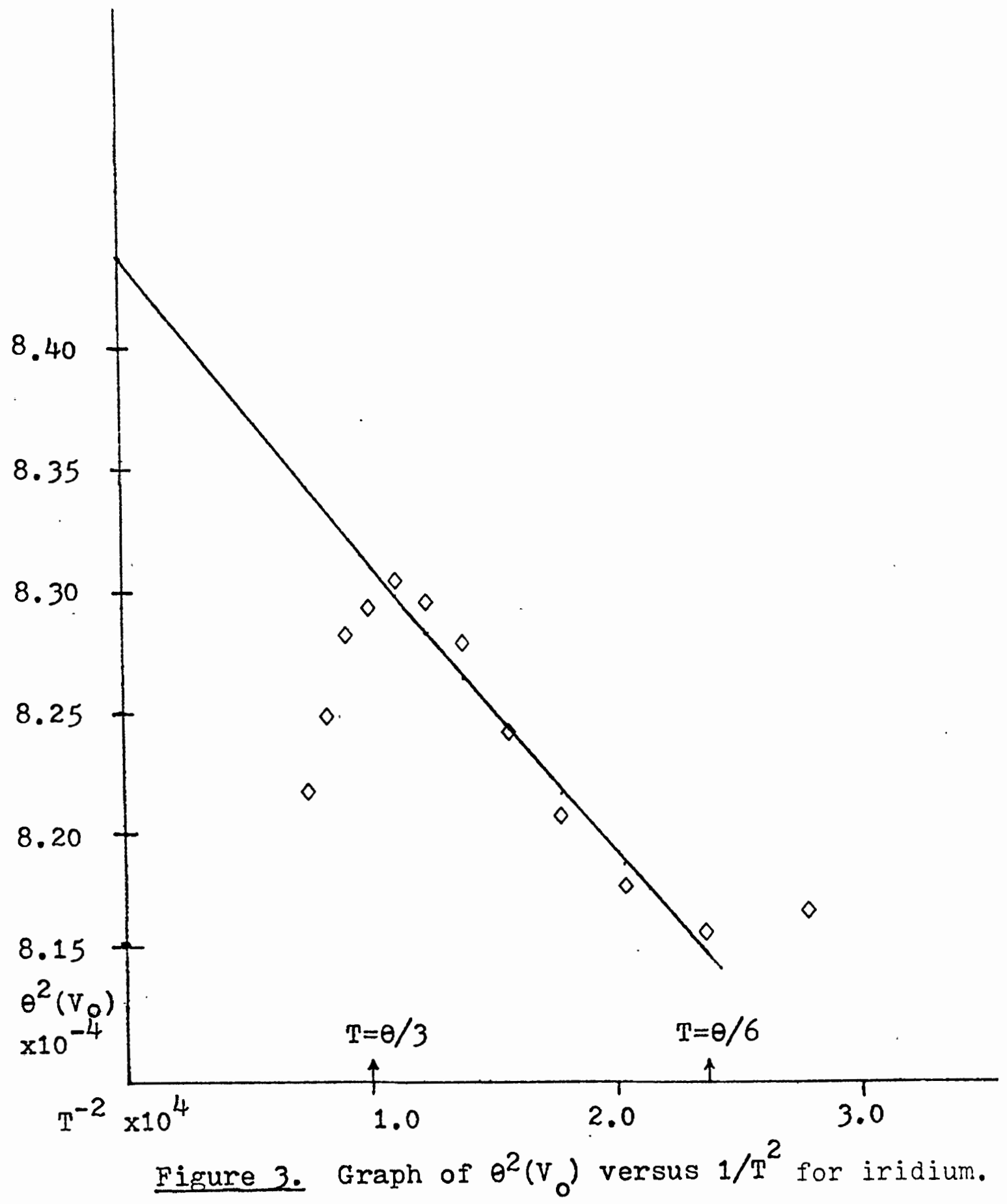


the Debye temperature. The initial slope of the curve fit to the data gives $\mathrm{A}_{\infty}^{4}$ and the curvature, the term in $1 / T^{4}$, gives $B \theta_{\infty}^{6}$. This enables one to write the host moments in terms of these coefficients;

$$
\begin{gathered}
u(2)=(3 / 5)(k / h)^{2} \theta_{\infty}^{2}, \\
u(4)=[(100 / 3) A+25 / 21] u(2)^{2}, \\
u(6)=(1400 B+100 A+125 / 81) u(2)^{3},
\end{gathered}
$$

It is important. to note that the temperature of the minimum in the Debye temperature (fig. 2) is approximately $\theta_{\infty} / 6$ and the maximum value reached occurs at a temperature around $\theta_{\infty} / 3$. Therefore by selecting only those points between the minimum and the maximum for the curve fit, one can make a meaningful extrapolation for the high temperature Debye theta, $\theta_{\infty}$. This $f i t$ is above the intermediate temperature range where the Debye temperature falls rapidly and below the temperature where anharmonicity causes the Debye temperature to fall off again. Woments and the frequency distribution. Since the moments are weighted integrals of powers of the frequency over the frequency distribution $G(\omega)$, eqn. (34), it will be instructive to make some observations about the frequency distribution for a generalized fcc structure (fig. 4).

In the Debye theory, the frequency distribution increases as the square of the frequency,

$$
G_{D}(\omega)=\left(3 / \omega_{m}^{3}\right) \omega^{2} \text {, }
$$

where the constant $\left(3 / \omega_{\mathrm{m}}^{3}\right)$ is obtained by normalizing the 


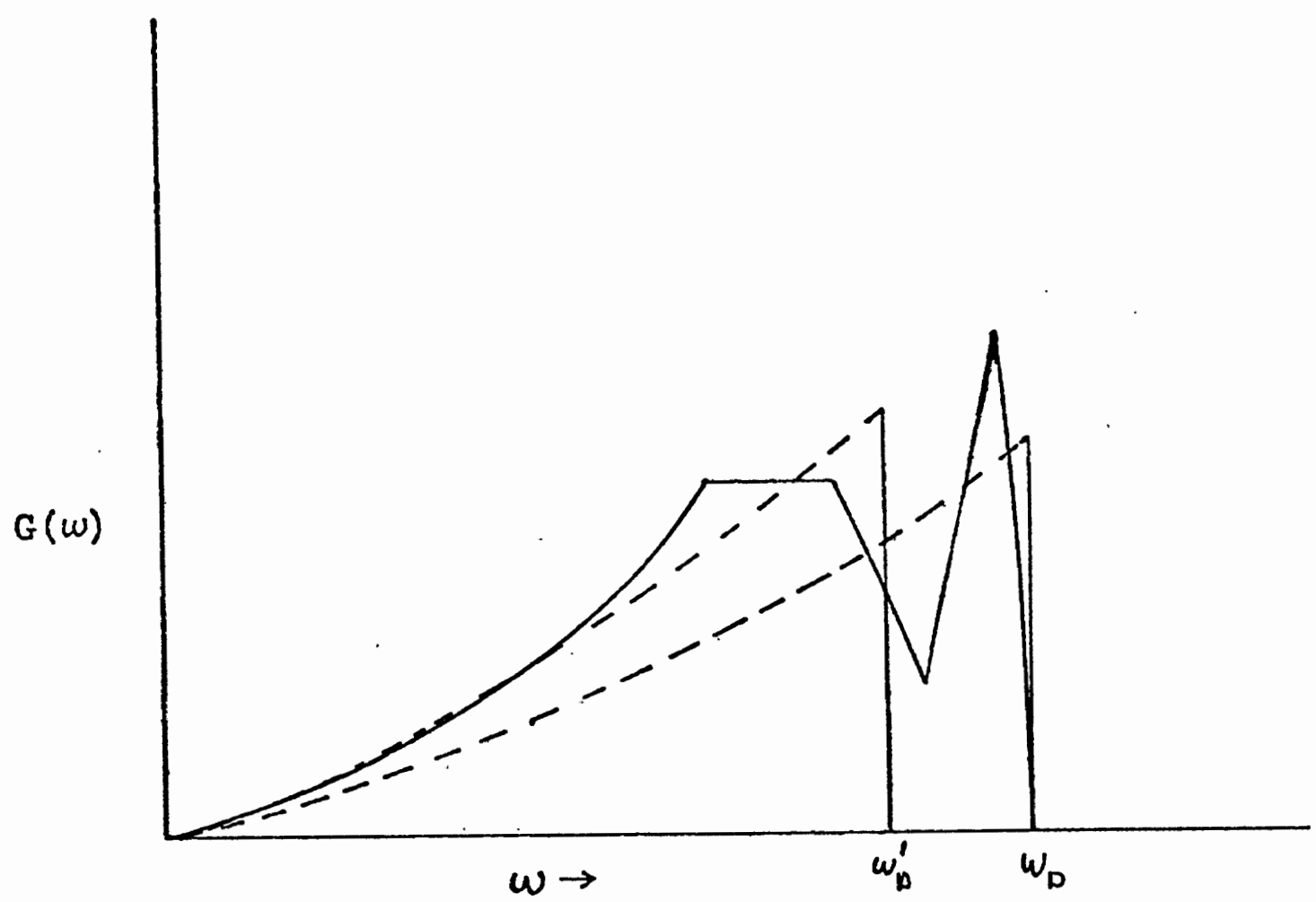

Figure 4. Generalized frequency distribution for a metal. 
integral as in eqn. (34), and $w_{m}$ is the limiting frequency of the integral (fig. 4).

If, however, the frequency spectrum has structure related to the higher order terms of eqn. (32), then the integral over the frequency spectrum will increase more rapidly, and the cutoff frequency will be correspondingly reduced ( $\omega_{\mathrm{m}}^{\prime}$ in fig. 4 ). It is this weighting of the higher order terms in the frequency distribution which characterizes the moments.

As the temperature increases from the low temperature region, the occupation of the transverse modes at lower frequencies increases rapidly until most of these modes are occupied. Then at the first singularity, (the edge of the plateau of the first peak in fig. 4), the density of states no longer increases rapidly as in the initial curve. In fact it is practically constant in the arbitrary distribution of fig. 4.

This allows the Debye temperature derived from the cutoff frequency to increase slowly as the temperature at which $\mathrm{c}_{\mathrm{v}}$ is determined increases. It is in this temperature region that one evaluates $\theta\left(V_{0}\right)$ in order to find the extrapolated high temperature $\theta_{\infty}$ which characterizes $u(2)$. As the temperature increases above $\theta_{0} / 3$, however, the lattice vibration can no longer be considered harmonic and $\theta$ derived from the heat capacity gradually falls off. This limits the number of useful data points in the 
analysis to the region approximately defined by $\theta / 6<\mathrm{T}<\theta / 3$. Therefore a statistical smoothing of the heat capacity data from very low to high temperatures is necessary to eliminate problems concerning the accuracy of the curve fit to $\theta^{2}\left(\mathrm{v}_{0}\right)$ in this region. 5 This increases the accuracy of the moments significantly.

In the determination of the moments for rhodium, an ambiguity developed in the initial three parameter curve fit. Here, the coefficients $A$ and $B$ yielded unexpected negative values, though very small. Forcing the data into an initial two parameter fit of the form;

$$
\theta^{2}=\theta_{\infty}^{2}-A \theta_{\infty}^{4} / T^{2} \text {, }
$$

allowed me to estimate a value of $\theta_{\infty}^{2}$ and $A$ to compare with the same values from the initial three parameter curve fit. By referring to table II we see that even this dramatic manipulation resulted in less than a half percent change in $u(2)$ and changes of 2 and 5 percent for $u(4)$ and $u(6)$ respectively. Even though these variations are at the limit of the resolution which can be carried through the calculations, they do not affect our primary intent here. For this work the fourth and sixth moments are only involved in the negative first and second moments, and in that case the difference is insignificant (table II).

Intermediate Temperature Region. We have until now only discussed the analysis of the heat capacity at high and low temperatures. Important information, and for the 
case of this work, the most useful information, can be obtained from the heat capacity at intermediate temperatures. In this range the anharmonic effects are small and the heat capacity is sensitive to the form of the frequency distribution.

Barron $^{2}$ has show that the expression for the thermal energy at moderately high temperatures can be used to obtain the first moment $u(1)$, which is directly related to the zero point energy of the crystal,

$$
\begin{array}{r}
E_{z}=3 / 2 N \hbar u(1)=3 R T\left(1+B_{2} u_{2}^{*} / 2 ! T^{2}-B_{4} u_{4}^{*} / 4 ! T^{4}+\right. \\
\left.B_{6} u_{6}^{*} / 6 ! T^{6}-\ldots\right)-\int_{0}^{T} C_{0} d T .
\end{array}
$$

Here $B_{2}, B_{4}$, and $B_{6}$ are the Bernoulli numbers, $u_{2}^{*}, u_{4}^{*}$, and $u_{6}^{*}$ are defined in eqn. (34), and $c_{0}$ is the heat capacity corrected to zero degree Kelvin. This correction is made by using the heat capacity at a given temperature to find the Debye temperature from standard tables of the Debye integral. 10 This Debye theta is corrected to zero degree by use of Grüneisen's constant, eqn. (26). This corrected Debye theta is then used in the standard table to find the corresponding heat capacity, now called $\mathrm{C}_{0}$.

Hwang $^{11}$ developed an expression for some moments using a similar integral form;

$$
\int_{0}^{\infty}\left(C_{0} / T^{n}\right) d T=3 R \Gamma(n+1) f(n) u_{1-n}^{*} \text {, }
$$

where $\Gamma(n)$ is the Gamma function, and $f(n)$ is the Riemann zeta function.

Therefore by integrating the Thirring ${ }^{8}$ expansion for 
$\mathrm{C}_{\mathrm{V}} / \mathrm{T}^{\mathrm{n}}$ and using eqn. (39), Barron derived an expression for the moments of the form: ${ }^{2}$

$$
\begin{aligned}
& u_{1-n}=(1 /(n+1) f(n))(\hbar / k)^{n-1}\left[(1 / 3 R) \int_{0}^{T}\left(C_{0} / T^{n}\right) d T+\right. \\
& \left(1 / \underline{T}^{n-1}\right)(1 / n-1)-\sum_{s=1}^{\infty}(-1)^{s+1}\left(B_{2 s} / 2 s i\right)\langle(2 s-1) /(2 s+n-1\rangle \\
& \left.(\hbar / k)^{2 s}\left(u(2 s) / \underline{T}^{2 s+n-1}\right)\right], \quad 1<n<4 .
\end{aligned}
$$

I in this equation is determined, essentially by trial and error, to be the temperature which causes the third term in the series of eqn. (40), i.e. $s=3$, to be less than one percent of the entire quantity in the square brackets. This allows convergence at the lowest temperature possible to minimize the influence of anharmonicity on the heat capacity. For the negative moments used here, $\underline{T}$ was taken to be $115 \mathrm{~K}$.

The integral was handled by using Stirling's method, and an initial guess at $\underline{\mathrm{T}}$ was used to set the limit of integration. It turned out that the initial guess for $\stackrel{m}{=}$ of $\theta / 3$ met the requirements for convergence on the first attempt. 


\section{EXPERIMENTAL PROCEDURE}

\section{PREPARATION OF MÖSSBAUER SOURCES}

The iridium Mössbauer source was prepared from a thin polycrystalline foil. The purity of the foil was 99.999 percent iridium. The foil was prepared by degreasing with acetone and alcohol, then washed with a weak (approximately 10\%) solution of $\mathrm{HCl}$ to clean and lightly etch the surface. The technique used to deposit the ${ }^{57}$ Co onto the surface was developed in this laboratory. When the 57 Co is produced at the accelerator, it is separated from the other products by using ion exchange resins. The ${ }^{57}$ Co activity comes out in a weakly acidic HCl solution which is dried in the shipping vial for transport to the laboratory. When it arrives, it is taken up in $95 \%$ ethanol in which it is slightly soluble. In order to prepare the source, the foil is heated to a modest temperature (on the order of 50 degrees Centigrade), then microliter quantities of the ethanol with the ${ }^{57}$ Co activity in solution are pipetted slowly onto a small spot. Since the ethanol will evaporate rapidly at this temperature, a very discrete source can be created, usually no more than $2 \mathrm{~mm}$ in diameter.

In order to have a reasonable count rate, at least 
one half millicurie of activity is deposited but not more than one millicurie. The iridium source used in this work contained .97 millicurie of 57 Co activity in a spot $2 \mathrm{~mm}$ in diameter before diffusion.

The diffusion of the ${ }^{57}$ Co activity into the interior of the sample is done at low pressure in a hydrogen atmosphere. The vacuum system was purged three times as the sample was warmed inside the furnace initially. Since the activity is present as the chloride salt, ${ }^{5} \mathrm{CoCl}$, the hydrogen is necessary to reduce the cobalt to a free metal at the surface with the HCl formed being pumped away. When the chamber temperature reached 700 degrees centigrade the $\mathrm{H}_{2}$ atmosphere was finally pumped away. Since iridium is a refractory metal with a melting point of 2683 degrees Kelvin, the furnace was allowed to rise to its maximum temperature of 1100 degrees centigrade in order to enhance the diffusion rate of ${ }^{57}$ Co into the Ir lattice. After 44 hours at this temperature, the sample was quenched by plunging the vycor tube with the iridium foil inside into a bucket of cold water. The initial cooling rate of the quenching procedure is on the order of several hundred degrees per second.

The iridium sample was then etched lightly with a $30 \% \mathrm{HCl}$ etching soution in order to remove any activity which remained on the surface. The total amount of activity in the sample after this cleaning was determined 
to be .78 millicurie.

An energy spectrum was then taken on a multichannel analyzer in order to determine that the activity had diffused to the interior of the sample.

The iridium source was then mounted on a velocity drive described by Brace. ${ }^{12}$ A Mössbauer velocity spectrum was taken and the results are in table III. The line shape of the source was slightly broader than the natural line shape $(.23 \mathrm{~mm} / \mathrm{sec}$ compared with $.20 \mathrm{~mm} / \mathrm{sec}$ ), but showed no irregularities, and based on past experience with other sources, it was assumed that the vast majority of the ${ }^{57}$ Co impurity atoms had found their way into substitutional sites in the iridium lattice.

\section{RECOIL FREE FRACTION}

In order to clarify the method by which the recoilfree fraction or $f$ is measured, a short explanation of the Mössbauer fraction is included here. For greater clarity, other sources should be consulted. 12

The Mössbauer effect allows one to observe the mean squared displacement of a gamma ray emitting impurity atom in a crystalline metal lattice. If the lifetime of the nuclear transition is long in relation to the period of the lattice vibration, (whioh is generally true), Iipkin 13 has shown that

$$
f=\exp \left[-k^{2}\left\langle x^{2}\right\rangle\right]
$$




\section{TABIE III}

VELOCITY SPECTRUM, IRIDIUM

\section{Peak 1}
Amplitude $(\%) \quad-7.90 \pm .18$
Position (mm/s) $\quad .528 \pm .001$
FWHM $(\mathrm{mm} / \mathrm{s}) \quad .226 \pm .001$
Peak 2

$\begin{array}{ll}\text { Amplitude }(\%) & -7.96 \pm .18 \\ \text { Position }(\mathrm{mm} / \mathrm{s}) & -1.121 \pm .001 \\ \text { FWHM }(\mathrm{mm} / \mathrm{s}) & .226 \pm .001\end{array}$


where $k$ is the gamma ray wave number, and $\left\langle x^{2}\right\rangle$ is the mean squared displacement of the emitting atom in the direction of emission of the gamma ray. When considering a very large population of emitting atoms, f can be interpreted as the fraction of gamma rays emitted without exciting a normal mode of lattice vibration due to recoil. Since there is no Doppler broadening of the gamma ray, these "resonant" gamma rays will be entirely absorbed by a "black wide absorber" 14 which is a chemical mixture of $57 \mathrm{Fe}$ salts whose line width is much wider (20 times) than the width (in energy) of the resonant gamma ray. Therefore one can count the total number of gamma rays emitted by shifting the energy of the resonant gamma ray out from under the absorber. That is done in this case by giving either source or absorber a high relative velocity with respect to the other. If the velocity drive is kept at rest, then the absorber will remove the resonant gamma rays and one can count the non-resonant gamma rays. Therefore the Mössbauer fraction is found experimentally as the difference of these two divided by the total;

$$
\begin{aligned}
f & =\frac{\text { to tal number of } 14.4 \mathrm{keV} \gamma \text {-rays }- \text { non-resonant } \gamma \text {-rays }}{\text { total number of } 14.4 \mathrm{keV} \gamma \cdot \text {-rays }} \\
& =\frac{\left(I_{\infty}-I_{B}\right)-\left(I_{0}-I_{B}\right)}{I_{\infty}-I_{B}}=\frac{\alpha I_{\infty}-I_{0}}{\alpha I_{\infty}^{\prime}-\beta I_{B}^{\prime}},
\end{aligned}
$$

where $I_{\infty}$ is the intensity of all the $14.4 \mathrm{keV}$ gamma rays, $I_{B}$ is the intensity of the background radiation which 
are within the energy range of the counting equipment, $I_{0}$ is the intensity of the non-resonant $14.4 \mathrm{keV}$ gamma rays, the primes indicate experimentally measurable quantities, and the correction factors $\alpha, \alpha^{\prime}$, and $\beta$ are determined ${ }^{12}$ to be: $\alpha=1.006, \alpha^{\prime}=1.009$, and $\beta=1.019$. This produces a raw $f$ value which is then corrected for the geometry of the experimental equipment and the "blackness" of the wide absorber to an absolute $f$ value. This involved multiplying the raw $f$ values by 1.032 for this experiment as set up on the velocity drive. Since the experinental procedure involved placing the source inside a cryostat and a furnace which both have different geometrical correction factors, a correction was made for that empirically by equating the $f$ values:oblained at room temperature in each to the $f$ value obtained on the velocity drive.

The method was the same as that described by Brace, but in this experiment, however, there was no problem with the blackness correction constant. Since the iridium source has an emission line close in energy to the center $o I$ the absorber, the thermal shift did not affect the value of the blackness correction constant.

The cryostat and the furnace were equipped with an electromechanical control which allowed the experimental temperature to be maintained to within one percent. A digital voltmeter was used with an appropriate thermocouple to accurately measure the temperature of the experiment. 
The rhodium source was prepared in essentially the same manner as I have described for iridium by Jim Grow. It had a narrow linewidth, and had already been characterized as a good source where the iron was a substitutional impurity in the rhodium lattice.

The combined systematic and statistical error in the measurement of the absolute $f$ value was kept under .5 percent of the f value by using a source with a sufficiently high count rate, and repeating the measurements approximately ten times. The absolute $f$ values and the negative logarithm used in finding the impurity moments are given in table IV. 
TABLE IV

RECOIL-FREE FRACTION $f$ AND LOG $f$ IRIDIUNI

RHODIUM

$\begin{array}{lllll}\text { Temp K } & f & -\log f & f & -\log f \\ 4 & .915 & .0888 & & \\ 20 & .914 & .0897 & & \\ 80 & .909 & .0953 & & \\ 130 & .893 & .1129 & & \\ 180 & .870 & .1396 & & \\ 230 & .848 & .1653 & .776 & .2541 \\ 295 & .808 & .2132 & .738 & .3045 \\ 350 & .785 & .2421 & .708 & .3447 \\ 400 & .760 & .2744 & .680 & .3868 \\ 450 & .737 & .3055 & .650 & .4302 \\ 500 & .712 & .3394 & .622 & .475 \\ 550 & .688 & .3743 & .596 & .518 \\ 600 & .663 & .4117 & & \end{array}$


IMPURITY MOMENTS AND EFFECTIVE FORCE CONSTANT RATIOS

As indicated in the section on the experimental method, the Mössbauer fraction can be expressed as

$$
f=\exp \left[-x^{2}<x^{2}\right] \text {. }
$$

or conversely,

$$
\left\langle x \xi^{2}=(-\ln f) / k^{2} .\right.
$$

From the analysis of Housley and Hess, ${ }^{1}$ one can show that the Thirring expansion for the energy of a crystal leads to an expression for the mean squared displacement in terms of the impurity moments. At high temperatures,

$$
\begin{aligned}
\langle\rangle_{T}^{2}=\left(k T / M^{\prime}\right) & {\left[\left(u \cdot(-2)+(1 / 12)(h / k T)^{2}-\right.\right.} \\
& \left.\left.(1 / 720)(h / k T)^{4} u \cdot(2)+\ldots\right)\right],
\end{aligned}
$$

and as $T$ goes to zero,

$$
\left\langle x_{0}^{2}=\left(h / 2 M^{\prime}\right) u^{\prime}(-1),\right.
$$

where $k$ is Boltzmann's constant, $M^{*}$ is the mass of the impurity atom, and the $u^{\prime}(n)$ are the impurity site moments as defined by Grow, Howard, Nussbaum, and Takeo. 16 However, let us observe here that these moments represent weishted frequencies over the integral of either the host or impurity response functions. For the host,

$$
u(n)=\int_{0}^{\infty} w^{n} G(w) d w, \quad u(0)=1,
$$

and for the impurity,

$$
u^{\prime}(n)=\int_{0}^{\infty} w^{n} G^{\prime}(w) d w, \quad u^{\prime}(0)=1
$$


Therefore, where the frequency distribution is unknown, these moments can be used to characterize the dynamical properties of the host or impurity. 16

Specifically, it has been shown ${ }^{1}$ that for harmonic cubic crystals in general

$$
u(2)=A / M \text {, }
$$

and for the impurity.

$$
u^{\prime}(2)=A^{\prime} / M^{\prime},
$$

where $M$ is the mass of the host, $M$ is the mass of the impurity, $A$ is known as the effective force constant of the host, and $A^{\cdot}$ is the effective force constant of the impurity. The ratio of these effective force constants can be expressed in an analytical form involving the host and impurity moments. 16

The impurity moments are determined (from eqns. 44, 45, and 46) to be, at high temperatures $(T<\theta / 2)$,

$$
u^{\prime}(-2)=M^{\prime}\left\langle x^{2} / k T-(1 / 12)(\hbar / k T)^{2},\right.
$$

and at Iiquid He temperatures,

$$
u \cdot(-1)=2 M \cdot / \hbar<x s^{2} \text {, }
$$

where all terms above the second on the right in eqn. (45) can be neglected. Also the host moments can be determined from eqn. (49) by use of neutron dispersion data, or as in this work, from heat capacity data.

Using Mannheim's impurity model and restricting it to the central force, nearest neighbors approximation, Grow, Howard, Nussbaum, and Takeo ${ }^{16}$ derived expressions 
for the effective force constant ratios; for $n=-2$,

$$
A / A^{\prime}=\left(1+\left(u^{\prime}(-2) M / u(-2) M \cdot-1\right) / B_{-2}\right), \quad(53)
$$

and for $n=-1$.

$$
\begin{gathered}
A / A^{\prime}=\left(1+\left(B_{4}\right)^{-1}\left(M / M^{\prime}\right)^{2 a}\left(u^{\prime}(-1) / u(-1)\left(M / M^{\prime}\right)^{\frac{1}{2}+a}-1\right)\right)^{2}, \\
a=\frac{1}{2}\left(\left(B_{-1}\right)^{-\frac{1}{2}}-1\right),
\end{gathered}
$$

where $u(n), u^{\prime}(n), M, M^{\prime}, A$, and $A^{\prime}$ are as defined previously and $B_{n}=u(2)^{n / 2} / u(n)$ is the ratio of the host moments to the $u(2)$ moment. This ratio is used because oi the model independent relation of $u(2)$ to the host restoring force, as in eqn. (49). 16

Just as for the host moments, we assume that the impurity moments are volume dependent. Therefore by evaluating $u^{\prime}(-2)$ at various temperatures and plotting the moments against $\Delta \mathrm{V} / \mathrm{V}$ from the linear expansion 17 $(\Delta V / V=3 \Delta I / I)$, we can obtain the value of $u^{\prime}(-2)$ in the quasiharmonic approximation from the intercept as $\Delta V / V$ goes to zero.

In table $V$ are listed the values for the effective force constant ratio $\mathrm{A} / \mathrm{A}^{\prime}$ derived from eqns. (53), and (54). Also included in the table are force constant ratios calculated from eqns. (53), and (54) using impurity moments determined from a recent report ${ }^{18}$ on the recoil free fraction, $f$, in fcc metals using ${ }^{119} \mathrm{Sn}$ as the impurity. 


\section{TABLE V}

IMPURITY MONENTS AND EFFECTIVE

\section{FORCE CONSTANT RATIOS}

$57_{\mathrm{Fe}}$ impurity system:

Ir host, $M / M^{\circ}=3.376, B_{-2}=.718, B_{-1}=.914 \quad B_{4}=.799$

$$
\begin{aligned}
& u(-1)=3.72 \times 10^{-14}(\mathrm{~s} / \mathrm{rad}) \\
& u^{\prime}(-1)=2.99 \times 10^{-14}(\mathrm{~s} / \mathrm{rad}) \\
& \mathrm{A}^{\prime} \mathrm{A}^{\prime}=2.61 \pm .43
\end{aligned}
$$

$u(-2)=1.60 \times 10^{-27}(\mathrm{~s} / \mathrm{rad})^{2}$

$u \cdot(-2)=8.37 \times 10^{-28}(\mathrm{~s} / \mathrm{rad})^{2}$

$\mathrm{A} / \mathrm{A}^{\circ}=2.07 \pm .24$

Rh host, $M / M^{\prime}=1.807, B_{-2}=.707$

$$
\begin{aligned}
& u(-2)=1.14 \times 10^{-27}(\mathrm{~s} / \mathrm{rad})^{2} \\
& u^{\prime}(-2)=1.04 \times 10^{-27}(\mathrm{~s} / \mathrm{rad})^{2} . \\
& A / A^{\prime}=1.92 \pm .23
\end{aligned}
$$

${ }^{119} \mathrm{Sn}$ impurity system:

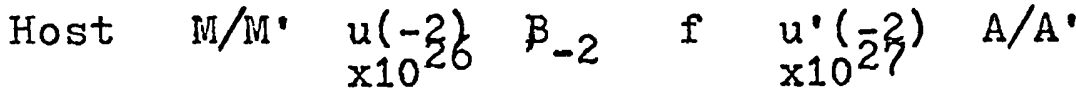

$\begin{array}{lllllll}\text { Rh } & .858 & .114 & .707 & .75 & .905 & .55\end{array}$

Ir $\quad \begin{array}{llllll}1.60 & .161 & .718 & .82 & .607 & .45\end{array}$ 


\section{DISCUSSION}

For ${ }^{57} \mathrm{Fe}$ in either Ir or $\mathrm{Rh}$, we see from table IV that the impurity-host restoring forces are smaller than the host-host restoring forces, since $A / A^{\prime}$ is greater than one. This is in contrast to the case of ${ }^{119} \mathrm{Sn}$ in $\mathrm{Ir}$ and $\mathrm{Rh}$ where the impurity-host restoring forces are larger than the host-host restoring forces since $A / A$ ' is less than one. The interatomic spacing for either $\operatorname{Ir}(3.84 \mathrm{~A})$ or $\mathrm{Rh}(3.80 \mathrm{~A})$ is slightly larger than the value for $\mathrm{Fe}(2.87 \mathrm{~A})$. Therefore the iron impurity will fit easily into a substitutional site in the lattice. On the other hand, the tin impurity is about twice the size of $\mathrm{Fe}$ with an interatomic spacing along the a axis of $5.82 \mathrm{~A}$. Because of this, one should expect more overlap between the orbitals of the tin impurity and the host than for the iron impurity. This would lead to larger interatomic forces for tin than for iron, and therefore smaller values of the effective force constant ratio.

For metals in general, the effective force constant ratios derived from low temperature $f$ values and heat capacity data agree with those derived from low temperature f values and neutron dispersion data. ${ }^{16}$ This demonstrates that the use of the heat capacity data, when combined with accurate Mössbauer $f$ values, can provide information about 
the host-impurity interaction, and is especially useful when the neutron dispersion relations are unknown. Such is the case for both Ir and Rh since the heat capacity data is well defined but neutron dispersion relations remain unknown.

For Ir, the effective force constant ratio determined from $u^{\prime}(-1) / u(-1)$ is twenty five percent higher than the value of $A / A^{\prime}$ determined from $u^{\prime}(-2) / u(-2)$. A similar pattern is exhibited by other $3 d, 4 d$, and $5 d$ transition elements, where the low temperature $f$ values yield systematically higher values of $A / A$ ' than the high temperature f measurements. ${ }^{16}$ This is paralleled to some extent by the relationship of the force constants found from neutron dispersion data. The value of $A / A$ ' determined from the low temperature $f$ values (i.e. $u^{\prime}(-1)$ ) and the negative moment, $u(-1)$, from neutron data is usually slightly larger than the value of $A / A^{\prime}$ determined from the high temperature $f$ values (i.e. $u^{\prime}(-2)$ ) and the negative moment $u(-2)$ determined from a host neutron density of states, $G(\omega)$, and eqn. (47). 16 Perhaps the anharmonicity at higher temperatures or the change of $G(\omega)$ with temperature are responsible for this effect.

By referring to table II, and recalling that $\beta_{n}=$ $u(2)^{n / 2} / u(n)$, we can make some casual observations about the structure of the frequency distribution, $G(\omega)$. The moment ratios for Ir and Rh are higher than for the other 
fcc metals. These higher values of $\beta_{4}, \beta_{-1}$, and $\beta_{-2}$ imply that the frequency spectrum is more compressed into the central frequencies than the other fcc metals. Hopefully this will be illuminated by neutron dispersion data. 


\section{BIBLIOGRAPHIY}

1. R. N. Housley and F. Hess, Phys. Rev. 146, 517 (1966).

2. T. H. K. Barron, W. T. Berg, and J. A. Morrison, Proc. R. Soc. Iond. A 242, 478 (1957).

3. P. D. Mannheim, Phys. Rev. 165, 1011 (1968).

4. M. W. Zemansky, Heat and Thermodynamics, fourth ed., (McGraw-Hill, New York, 1957).

5. G. T. Furukawa, M. I. Reilly, and J. S. Gallagher, J. Phys. Chem. Ref. Data 3, 197 (1974).

6. C. Kittel, Introduction to Solid State Physics, 2nd ed., (John Wiley, New York, 1953).

7. B. Yates, Thermal Expansion, (Plenum, New York, 1972).

8. H. Thirring, Phys. Z. 14, 867 (1913).

9. C. Domb and I. Salter, Phil. Mag. 43, 1083 (1952).

10. J. E. Kilpatrick and R. H. Sherman, Tables of Debye Integrals, (AEC, Los Alamos, 1964).

11. J. I. Hwang, J. Chem. Phys. 22, 154 (1954).

12. F. Brace, M. S. Thesis, (PSU, 1972).

13. H. S. Lipkin, Ann. Phys. 2, 332 (1960).

14. J. G. Dash, Mössbauer Effect Methodology, vol. 3. (New England Nuclear Corporation, 1970).

15. K. A. Gschneidner, Solid State Physics, vol. 16, 276 (1964).

16. J. M. Grow, D. G. Howard, R. H. Nussbaum, and $\mathbb{V}_{1}$. Takeo, Phys. Rev. B, 17, 15 (1978).

17. Y. S. Touloukian and E. H. Buyco, Thermophysical Properties of Metals, vol. 12 (Plenum, New York, 1975).

18. A. N. Larsen and G. Weyer, J. Phys. F: Metal Phys., 2. 27 (1979). 